COMMENT. Adolescents with or without ADHD have a similar risk of psychoactive substance use disorders (PSUD), with a frequency of $15 \%$. However, children with ADHD may be at higher risk for early-onset addictions than controls. PSUD in adolescents is associated with a history of conduct and bipolar disorders, but not oppositional defiant disorder, major depression, or anxiety disorders. The risk of PSUD is increased in adults with ADHD, and a sharp increase in PSUD may be expected in adolescent ADHD subjects as they become adults.

Comorbid ADHD and substance abuse. Comorbidity of ADHD among adolescents in treatment for substance abuse is reported from the National Center for Post Traumatic Stress Disorder, Veteran's Affairs Medical Center, West Haven, CT. (Horner BR, Scheibe KE. Prevalence and implications of attention-deficit hyperactivity disorder among adolescents in treatment for substance abuse. L Am Acad Child Adolesc Psychiatry Jan 1997;36:30-36). Attempts to self-medicate symptoms of ADHD may underlie substance use and abuse, especially in previously untreated adolescents. Prescribed medical treatment for ADHD may decrease drug craving in adults with ADHD and improve functioning.

ADHD and cigarette smoking. ADHD, particularly when associated with conduct disorders, is a significant risk factor for early cigarette smoking in children and adolescents in a study at the Massachusetts General Hospital, Boston. (Milberger S, Biederman J, Faraone SV, Chen L, Jones J. ADHD is associated with early initiation of cigarette smoking in children and adolescents. IAm Acad Child Adolesc Psychiatry Jan 1997;36:37-44).

\title{
ADHD AND MOTOR VEHICLE SKILIS AND RISKS
}

The motor vehicle skills, risks, and accidents in 25 young adults with ADHD were compared with 23 non-ADHD adult control subjects, aged 17 to 30 years, at the Departments of Psychiatry and Neurology, University of Massachusetts Medical Center, Worcester, MA. ADHD young adults were twice as likely to be cited for unlawful speeding, had more crashes, more accidents involving bodily injury, more likely to have licenses suspended, and poorer driving habits and performance despite adequate driving knowledge, when compared to controls. (Barkley RA, Murphy KR, Kwasnik D. Motor vehicle driving competencies and risks in teens and young adults with attention deficit hyperactivity disorder. Pediatrics Dec 1996;98:1089-1095). (Reprints: Russell A Barkley PhD, Department of Psychiatry, University of Massachusetts Medical Center, 55 Lake Ave North, Worcester, MA 01655).

COMMENT. This study confirms previous reports of an increased risk of motor vehicle accidents and injury among ADHD young adults. The findings indicate the need for continued treatment and supervision of adolescents with ADHD into adulthood, particularly in relation to self-regulation of motor control and lessening of impulsive behavior.

\section{METHYLPHENIDATE USAGE FOR ADHD IN THE 1990s}

The usage and usage trends for methylphenidate (MPH) treatment of ADHD from 1990 through 1995 were estimated by the Departments of Psychiatry and Pediatrics, Johs Hopkins University School of Medicine, Baltimore County Health Department, and the Schools of Pharmacy and Medicine, University of Maryland, Baltimore, MD. A review of regional and national databases showed a 2.5 -fold increase in the prevalence of MPH treatment of youths with $\mathrm{ADD}$ in this time period. Approximately $2.8 \%(1.5$ 
million) US children aged 5 to 18 received MPH for ADD in 1995 . The increased usage was related to more prolonged treatment, more girls, and adolescents receiving medication for ADD. (Safer DJ, Zito JM, Fine EM. Increased methylphenidate usage for attention deficit disorder in the 1990s. Pediatrics Dec 1996;98:1084-1088). (Reprints: Daniel J Safer MD, 7702 Dunmanway, Dundalk, MD 21222).

COMMENT. A 2.8-fold increase in methylphenidate usage between 1990 and 1995, as shown in this study, is far less than the media claims of a 6-fold increased usage, based on DEA production quotas for methylphenidate in this time period. The findings do not address the appropriateness of stimulant therapy for ADHD.

\section{SLEEP DISORDERS IN NEUROLOGICAL PRACTICE}

Pediatric sleep disorders are reviewed from the Infant and Family Development Laboratory, Department of Psychiatry and Graduate Program in Human Development, University of California, Davis. Sleep disorders associated with neurological disorders include: 1) sleep-related epilepsy; 2) sleep-related headaches; 3) degenerative disorders; 4) developmental disorders (Down syndrome; Prader-Willi syndrome); 5) methylphenidate treatment for ADHD causing sleep disturbance; and 6) Kleine-Levin syndrome, with episodic excessive somnolence. Medical and psychiatric disorders that interfere with sleep include: asthma, gastroesophageal reflux, psychoses, mood disorders, anxiety, and substance abuse disorders. Primary sleep disorders include dyssomnias (obstructive sleep apnea with enlarged tonsils and adenoids, narcolepsy, extrinsic sleep irregularities of infants, circadian rhythm sleep disorders); parasomnias (sleep terrors, sleepwalking, head banging, rocking, talking, leg cramps, nightmares, bruxism, enuresis). (Anders TF, Eiben LA. Pediatric sleep disorders; a review of the past 10 years. I Am Acad Child Adolesc Psychiatry Jan 1997;36:9-20). (Reprints: Dr Anders, Department of Psychiatry, UCDMC, 4430 V Street, Sacramento, CA 95817).

COMMENT. The pediatric neurologist may be consulted because of REM parasomnias and the differentiation of nightmares, sleep terrors, and nocturnal frontal lobe seizures. EEGs are frequently nonspecific, and videopolysomnographic monitoring is often necessary. A trial of anticonvulsant medication, eg clonazepam, may be warranted in suspected but unconfirmed cases of seizures. (see Ped Neur Briefs Oct 1996 for reports of nocturnal frontal lobe epilepsy and differential diagnosis).

\section{LANGUAGE DISORDERS}

\section{ACQUIRED NEUROLOGIC MUTISM}

The behavioral features of four children with acquired neurologic mutism are reported from the Department of Neurology, University Hospital Rotterdam-Dijkzigt, Rotterdam; and Department of Medical Psychology, Ziekenhuis Walcheren, Vlissingen, The Netherlands. Neuropsychologic examinations revealed changes in phonation, orofacial movements, communicative behavior, and linguistic functions. Case 1, akinetic mutism, post severe head injury, hemorrhage in left fronto-parietal area, spastic quadriplegia, motionless except for slow eye movements and head turning when addressed, no orofacial movements except smacking of lips, recovered 